\title{
Article \\ Comparison of Ameliorative Effects between Probiotic and Biodegradable Bacillus subtilis on Zearalenone Toxicosis in Gilts
}

\author{
Wenqiang Shen ${ }^{1,+}\left(\mathbb{D}\right.$, Yaojun Liu ${ }^{1,+}{ }^{,}$Xinyue Zhang ${ }^{1}$, Xiong Zhang ${ }^{1}$, Xiaoping Rong ${ }^{1}$, Lihong Zhao ${ }^{1}$, Cheng Ji ${ }^{1}$, \\ Yuanpei Lei ${ }^{1}$, Fengjuan $\mathrm{Li}^{2}$, Jing Chen ${ }^{2}$ and Qiugang Ma ${ }^{1, * \mathbb{D}}$ \\ 1 State Key Laboratory of Animal Nutrition, China Agricultural University, Beijing 100193, China; \\ Shenwenqiang1996@163.com (W.S.); yaojunliu@outlook.com (Y.L.); zhangxinyue090@163.com (X.Z.); \\ Zhangxiongcau@163.com (X.Z.); rxp409291@163.com (X.R.); zhaolihongcau@cau.edu.cn (L.Z.); \\ jicheng@cau.edu.cn (C.J.); lypcau@126.com (Y.L.) \\ 2 FuQing Fengze Agricultural Science and Technology Development Co., Ltd., Fuzhou 350011, China; \\ lifengjuan-1986@163.com (F.L.); chenjing101488@163.com (J.C.) \\ * Correspondence: maqiugang@cau.edu.cn \\ + These authors contributed equally to this work.
}

check for updates

Citation: Shen, W.; Liu, Y.; Zhang, X.; Zhang, X.; Rong, X.; Zhao, L.; Ji, C.;

Lei, Y.; Li, F.; Chen, J.; et al.

Comparison of Ameliorative Effects between Probiotic and Biodegradable Bacillus subtilis on Zearalenone

Toxicosis in Gilts. Toxins 2021, 13, 882. https: / / doi.org/10.3390/

toxins13120882

Received: 16 November 2021

Accepted: 8 December 2021

Published: 10 December 2021

Publisher's Note: MDPI stays neutral with regard to jurisdictional claims in published maps and institutional affiliations.

Copyright: (c) 2021 by the authors. Licensee MDPI, Basel, Switzerland. This article is an open access article distributed under the terms and conditions of the Creative Commons Attribution (CC BY) license (https:/ / creativecommons.org/licenses/by/ $4.0 /)$.

\begin{abstract}
This study was conducted to compare the potential ameliorative effects between probiotic Bacillus subtilis and biodegradable Bacillus subtilis on zearalenone (ZEN) toxicosis in gilts. Thirty-six Landrace $\times$ Yorkshire gilts (average BW $=64 \mathrm{~kg}$ ) were randomly divided into four groups: (1) Normal control diet group (NC) fed the basal diet containing few ZEN (17.5 $\mu \mathrm{g} / \mathrm{kg}$ ); (2) ZEN contaminated group (ZC) fed the contaminated diet containing an exceeded limit dose of ZEN (about $300 \mu \mathrm{g} / \mathrm{kg}$ ); (3) Probiotic agent group (PB) fed the ZC diet with added $5 \times 10^{9} \mathrm{CFU} / \mathrm{kg}$ of probiotic Bacillus subtilis ANSB010; (4) Biodegradable agent group (DA) fed the ZC diet with added $5 \times 10^{9} \mathrm{CFU} / \mathrm{kg}$ of biodegradable Bacillus subtilis ANSB01G. Results showed that Bacillus subtilis ANSB010 and ANSB01G isolated from broiler intestinal chyme had similar inhibitory activities against common pathogenic bacteria. In addition, the feed conversion ratio and the vulva size in DA group were significantly lower than ZC group $(p<0.05)$. The levels of IgG, IgM, IL-2 and TNF $\alpha$ in the ZC group were significantly higher than PB and DA groups $(p<0.05)$. The levels of estradiol and prolactin in the ZC group was significantly higher than those of the NC and DA groups $(p<0.05)$. Additionally, the residual ZEN in the feces of the ZC and PB groups were higher than those of the $\mathrm{NC}$ and DA groups $(p<0.05)$. In summary, the ZEN-contaminated diet had a damaging impact on growth performance, plasma immune function and hormone secretion of gilts. Although probiotic and biodegradable Bacillus subtilis have similar antimicrobial capacities, only biodegradable Bacillus subtilis could eliminate these negative effects through its biodegradable property to ZEN.
\end{abstract}

Keywords: zearalenone; degradable Bacillus subtilis; probiotic Bacillus subtilis; gilts

Key Contribution: The alleviating effects of biodegradable Bacillus subtilis ANSB01G on the ZENpoisoned gilts were compared to probiotic Bacillus subtilis ANSB010, which was isolated from the same source and with similar bacteriostatic activity. Results showed that the improvement of ANSB01G on ZEN-poisoned gilts comes from its biodegradation activity to ZEN; not from its antibacterial activity.

\section{Introduction}

Zearalenone (ZEN), known as an F-2 mycotoxin, is a powerful estrogenic metabolite produced by certain species of Fusarium and Erysipelas spp. [1]. Several results showed that many feedstuffs for animals have been seriously contaminated with ZEN around the world [2,3], which could cause hyperestrogenism and fertility disorder in sows [4]. Moreover, a previous study found that exposure of post-weaning gilts to ZEN could increase the oxidative stress and had a negative impact on genital organs [5]. In addition, 
recent study found that ZEN can interfere with immune mediators at the spleen level and induce an intense inflammatory response [6]. Therefore, it is critical to find the appropriate and effective detoxifying strategies to prevent contamination by ZEN in animal husbandry.

Numerous researches have reported that physical, chemical and biological methods can be used to counter mycotoxicosis [7-9]. However, most of these methods are impractical or potentially unsafe because of losses in the nutritional value, high equipment costs and formation of toxic residues or derivatives [10]. Biodegradation is eco-friendly and highly efficient in minimizing the harmfulness of mycotoxins in feeds $[11,12]$. Previous work from our laboratory reported that Bacillus subtilis ANSB01G, which has both a biodegradable effect against ZEN and probiotic activities against pathogenic bacteria, can alleviate toxicosis of ZEN in pre-pubertal female gilts $[13,14]$. However, the article did not clarify whether the reduced toxicity was due to its probiotic or biodegradable properties.

Therefore, the aim of this study was to investigate the effects of biodegradable and probiotic Bacillus subtilis on growth performance, serum biochemical indexes and hormone, serum antioxidant, immune indicators and mycotoxin residue in gilts exposed to ZEN for $25 \mathrm{~d}$ in vivo, as well as the inhibitory activity of common harmful bacteria in vitro.

\section{Results}

2.1. Biochemical and Physiological Characteristics of Bacillus subtilis ANSB010 and ANSB01G

The colony morphologies showed that the surfaces of Bacillus subtilis ANSB010 and ANSB01G colonies are rough, opaque and milky white (Figure 1A,B). Under the microscope, the cells were found to be short, thin rods, positive for Gram staining and capable of forming spores (Figure 1C,D). As shown in Table 1, the physiological and biochemical results revealed ANSB010 and ANSB01G had typical characteristics of Bacillus spp., such as growing well at $37^{\circ} \mathrm{C}$ but not at 10 and $55^{\circ} \mathrm{C}$; and being able to utilize cellulose, glucose and maltose as the only carbon source. Moreover, a phylogenetic tree based on $16 \mathrm{~s}$ rDNA sequences suggested that both ANSB010 and ANSB01G have a close evolutionary relationship to Bacillus subtilis (Figure 1E).

A

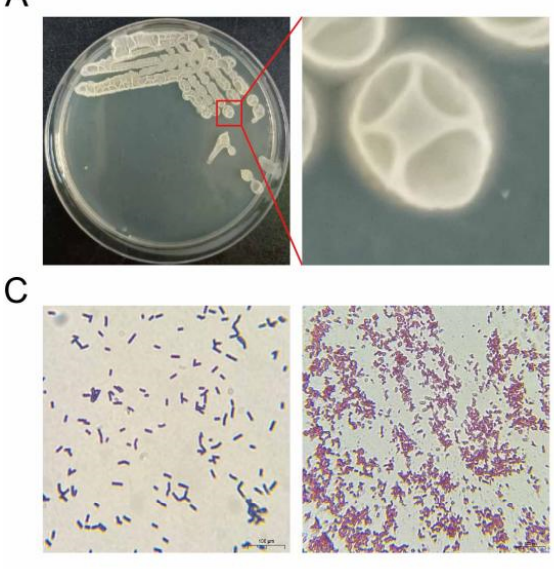

B
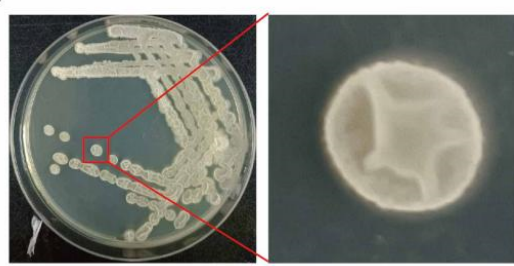

D

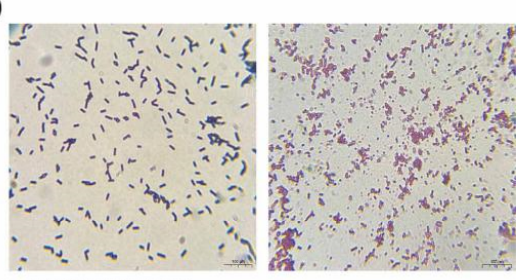

Bacillus subtilis (KX768309.1)

${ }_{00}$ Bacillus subtilis ANSB01G

Bacillus subtilis ANSB010

Bacillus subtilis (OK335854.1)

Bacillus coagulans (MN890027.1)

Halobacillus halophilus (LC654177.1)

Anoxybacillus flavithermus (GQ342689.1)

Geobacillus stearothermophilu (KX674365.1)

100 Lactococcus garvieae (NR152050.1)

Lactococcus garvieae (LT631769.1)

Bacillus pumilus (MF692772.1)

Figure 1. Colony characteristics of ANSB010 (A) and ANSB01G (B); cell (left) and spore (right) morphology of ANSB010 (C) and ANSB01G (D), scale bar, $100 \mu \mathrm{m}$; (E) the phylogenetic tree of Bacillus subtilis ANSB010 and ANSB01G, the GenBank accession numbers of sequences are shown in round brackets. 
Table 1. Biochemical and physiological characteristics of Bacillus subtilis ANSB010 and ANSB01G.

\begin{tabular}{|c|c|c|c|c|c|}
\hline Experimental Projects & ANSB010 $^{1}$ & ANSB01G $^{2}$ & Experimental Projects & ANSB010 & ANSB01G \\
\hline Gram & + & + & Glucose & + & + \\
\hline Cell shape & Rod-shape & Rod-shape & Maltose & + & + \\
\hline Cell diameter $>1 \mu \mathrm{m}$ & + & + & Sucrose & + & + \\
\hline Spore forming & + & + & D-xylose & + & + \\
\hline Spore dilation & - & - & L-xylose & - & - \\
\hline Round spores & - & - & D-arabinose & - & - \\
\hline Glycerol & + & + & L-arabinose & + & + \\
\hline Cellulose utilization & + & + & D-mannitol & + & + \\
\hline Catalase & + & + & Gas production using glucose & - & - \\
\hline Oxidase test & + & + & Citrate utilization & + & + \\
\hline Anaerobic & - & - & Growth at 10 or $50^{\circ} \mathrm{C}$ & - & - \\
\hline Voges-Proskauer (VP) test & + & + & Growth at $37^{\circ} \mathrm{C}$ & + & + \\
\hline $\mathrm{VP}<\mathrm{pH} 6$ & + & + & Growth at pH 5.7 & + & + \\
\hline $\mathrm{VP}>\mathrm{pH} 7$ & - & - & Growth on $7 \% \mathrm{NaCl}$ & + & + \\
\hline Methyl red test & - & - & Hydrolysis of starch & + & + \\
\hline Gluconate & - & - & Decomposition of casein & + & + \\
\hline Xylitol & - & - & Nitrate reduction & + & + \\
\hline
\end{tabular}

${ }^{1}{ }^{\prime}+,{ }^{\prime},-$ ' and ' $w$ ' mean positive, negative and weak response, respectively. ${ }^{2}$ The results of ANSB01G after domestication were consistent with Lei et al. [13].

\subsection{Bacteriostatic and ZEN-Degrading Effects of ANSB010 and ANSB01G}

As shown in Figure 2 and Table 2, probiotic Bacillus subtilis ANSB010 and biodegradable Bacillus subtilis ANSB01G have a visible antibacterial effect on Escherichia coli (E. coli), Salmonella choleraesuis (S. choleraesuis) and Staphylococcus aureus (S. aureus) compared to the control group (Con) $(p<0.05$, Table 2), while there was no significant difference in the antibacterial effect between ANSB010 and ANSB01G ( $p>0.05$, Table 2). Importantly, we noticed that ANSB01G could degrade $65.13 \%, 92.57 \%$ and $100.00 \%$ of ZEN in the fermentation broth at $6 \mathrm{~h}, 24 \mathrm{~h}$ and $48 \mathrm{~h}$, respectively, but ANSB010 could not $(p<0.05$, Table 3). Additionally, Supplementary Material Figures S1 and S2 show the representative chromatograms of degradation tests.

A

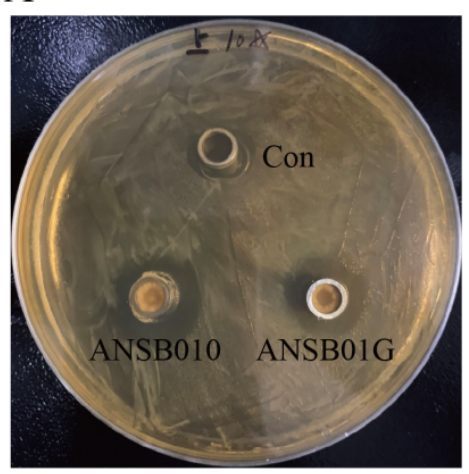

E. coli
B

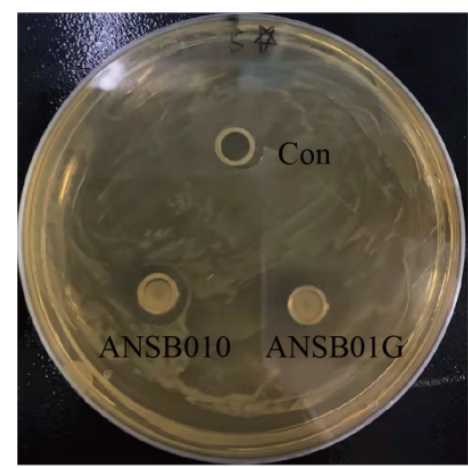

S. choleraesuis

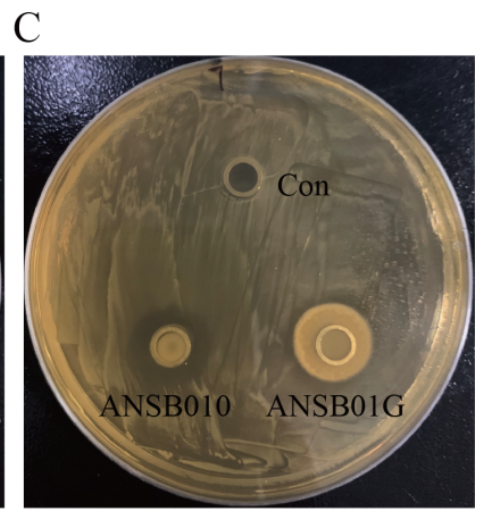

S. aureus

Figure 2. The antibacterial effects of probiotic Bacillus subtilis ANSB010 and biodegradable Bacillus subtilis ANSB01G on E. coli (A), S. choleraesuis (B) and S. aureus (C). E. coli, Escherichia coli; S. choleraesuis, Salmonella choleraesuis; S. aureus, Staphylococcus aureus. Con: MRS medium; ANSB010, probiotic Bacillus subtilis ANSB010; ANSB01G, biodegradable Bacillus subtilis ANSB01G. 
Table 2. Antibacterial effects of Bacillus subtilis ANSB010 and ANSB01G.

\begin{tabular}{cccccc}
\hline \multirow{2}{*}{$\begin{array}{c}\text { Indicator } \\
\text { Bacteria }\end{array}$} & \multicolumn{2}{c}{ Antibacterial Circle Diameter (cm) } & \multirow{2}{*}{ SEM } & \multirow{2}{*}{$\boldsymbol{p}$-Value } \\
\cline { 2 - 3 } & Con & ANSB010 & ANSB01G & & \\
\hline E. coli & $1.10^{\mathrm{b}}$ & $1.76^{\mathrm{a}}$ & $1.68^{\mathrm{a}}$ & 0.07 & 0.00 \\
S. choleraesuis & $0.86^{\mathrm{b}}$ & $1.62^{\mathrm{a}}$ & $1.71^{\mathrm{a}}$ & 0.09 & 0.00 \\
S. aureus & $1.07^{\mathrm{b}}$ & $1.74^{\mathrm{a}}$ & $1.71^{\mathrm{a}}$ & 0.08 & 0.00 \\
\hline
\end{tabular}

Con: MRS medium; ANSB010, probiotic Bacillus subtilis ANSB010; ANSB01G, biodegradable Bacillus subtilis ANSB01G. Different superscript letters represent significant difference.

Table 3. The degradation rate (\%) of ANSB010 and ANSB01G on zearalenone in fermentation medium.

\begin{tabular}{ccccc}
\hline Time & ANSB010 & ANSB01G & SEM & $p$-Value \\
\hline $6 \mathrm{~h}$ & $0.05^{\mathrm{b}}$ & $65.13^{\mathrm{a}}$ & 12.23 & 0.00 \\
$24 \mathrm{~h}$ & $0.38^{\mathrm{b}}$ & $92.57^{\mathrm{a}}$ & 4.81 & 0.00 \\
$48 \mathrm{~h}$ & $-0.26^{\mathrm{b}}$ & $100.00^{\mathrm{a}}$ & 4.22 & 0.00
\end{tabular}

ANSB010, probiotic Bacillus subtilis ANSB010; ANSB01G, biodegradable Bacillus subtilis ANSB01G. Different superscript letters represent significant difference.

\subsection{Growing Performance}

As shown in Table 4, no significant difference were observed for the initial weight, terminal weight and average daily gain (ADG) $(p>0.05)$, while there was a decreasing trend of average daily feed intake (ADFI) in probiotic Bacillus subtilis ANSB010 agent (PB) and biodegradable Bacillus subtilis ANSB01G agent (DA) groups $(p=0.10)$ compared to ZEN contaminated (ZC) group. Of the four groups, the ZC group had the highest feed conversion ratio $(\mathrm{F} / \mathrm{G})$ value $(p<0.05)$, and was 1.07 - and 1.13 -fold higher than the PB and DA group, respectively.

Table 4. Comparison of growth performance among different treatment groups.

\begin{tabular}{ccccccc}
\hline Items & NC & ZC & PB & DA & SEM & $p$-Value \\
\hline Initial weight, kg & 63.43 & 64.14 & 64.11 & 64.4 & 1.78 & 0.99 \\
Terminal weight, kg & 84.05 & 84.98 & 85.12 & 86.00 & 1.72 & 0.93 \\
ADG, g & 825 & 834 & 841 & 864 & 29.69 & 0.85 \\
ADFI, g & 2151 & 2244 & 2114 & 2047 & 81.44 & 0.10 \\
F/G & $2.61^{\mathrm{abc}}$ & $2.69^{\mathrm{ab}}$ & $2.52^{\mathrm{bc}}$ & $2.38^{\mathrm{c}}$ & 0.08 & $<0.01$
\end{tabular}

ADG, average daily gain; ADFI, average daily feed intake; $\mathrm{F} / \mathrm{G}$, feed conversion ratio; $\mathrm{NC}$, normal control diet group; ZC, Zearalenone (ZEN)-contaminated group; PB, probiotic Bacillus subtilis group; DA, biodegradable Bacillus subtilis group. Different superscript letters represent significant difference.

\subsection{Vulva Size}

The effects of the four diets on the vulva size are shown in Table 5 . The vaginal length and area of the DA group was significantly lower compared to the ZC group $(p<0.05)$, and there was no significant difference between the ZC and PB groups $(p>0.05)$. Interestingly, there was a decreasing trend of vaginal width among four groups $(p=0.10)$. The vaginal volume of the DA group was dramatically lower compared to all other groups $(p<0.05)$, and that of the PB group was significantly lower than that of the ZC group $(p<0.05)$, but no significant difference existed between the $\mathrm{ZC}$ and normal control diet (NC) groups, or PB and NC groups $(p>0.05)$. There was no significant difference in the vaginal height among the four groups $(p>0.05)$.

\subsection{Serum Biochemical Indicators, Antioxidant and Immunology Parameters}

There was no significant difference in the serum biochemical indicators (e.g., total protein (TP), albumin (ALB), alkaline phosphatase (ALP), aspartate aminotransferase (AST), alanine aminotransferase (ALT), creatinine (CRE) and urea nitrogen (BUN)) among these groups (Figure $3 \mathrm{~A}-\mathrm{G}, p>0.05$ ), nor in the levels of glutathione peroxidase (GSHPx) (Figure 4A, $p>0.05$ ) and malondialdehyde (MDA) (Figure 4C, $p>0.05$ ), which were 
indicators of antioxidant activities. Interestingly, the level of superoxide dismutase (SOD) in the PB group was significantly lower than that of the ZC group (Figure $4 \mathrm{~B}, p<0.05$ ).

Table 5. The values of vulva indexes in different treatment groups.

\begin{tabular}{ccccccc}
\hline Items & NC & ZC & PB & DA & SEM & $p$-Value \\
\hline Length, cm & $3.12^{\mathrm{ab}}$ & $3.34^{\mathrm{a}}$ & $2.93^{\mathrm{ab}}$ & $2.85^{\mathrm{b}}$ & 0.66 & 0.03 \\
Width, cm & 2.51 & 2.53 & 2.42 & 2.21 & 0.48 & 0.10 \\
Height, cm & 2.36 & 2.30 & 1.90 & 1.95 & 0.75 & 0.43 \\
Area, cm & $2.15^{\mathrm{ab}}$ & $6.64^{\mathrm{a}}$ & $5.58^{\mathrm{ab}}$ & $4.99^{\mathrm{b}}$ & 0.21 & 0.03 \\
Volume, & $4.83^{\mathrm{ab}}$ & $5.11^{\mathrm{a}}$ & $3.54^{\mathrm{b}}$ & $3.23^{\mathrm{c}}$ & 0.24 & 0.00 \\
$\mathrm{~cm}^{3}$ & & & &
\end{tabular}

NC, normal control diet group; ZC, ZEN-contaminated group; PB, probiotic Bacillus subtilis group; DA, biodegradable Bacillus subtilis group. Different superscript letters represent significant difference.
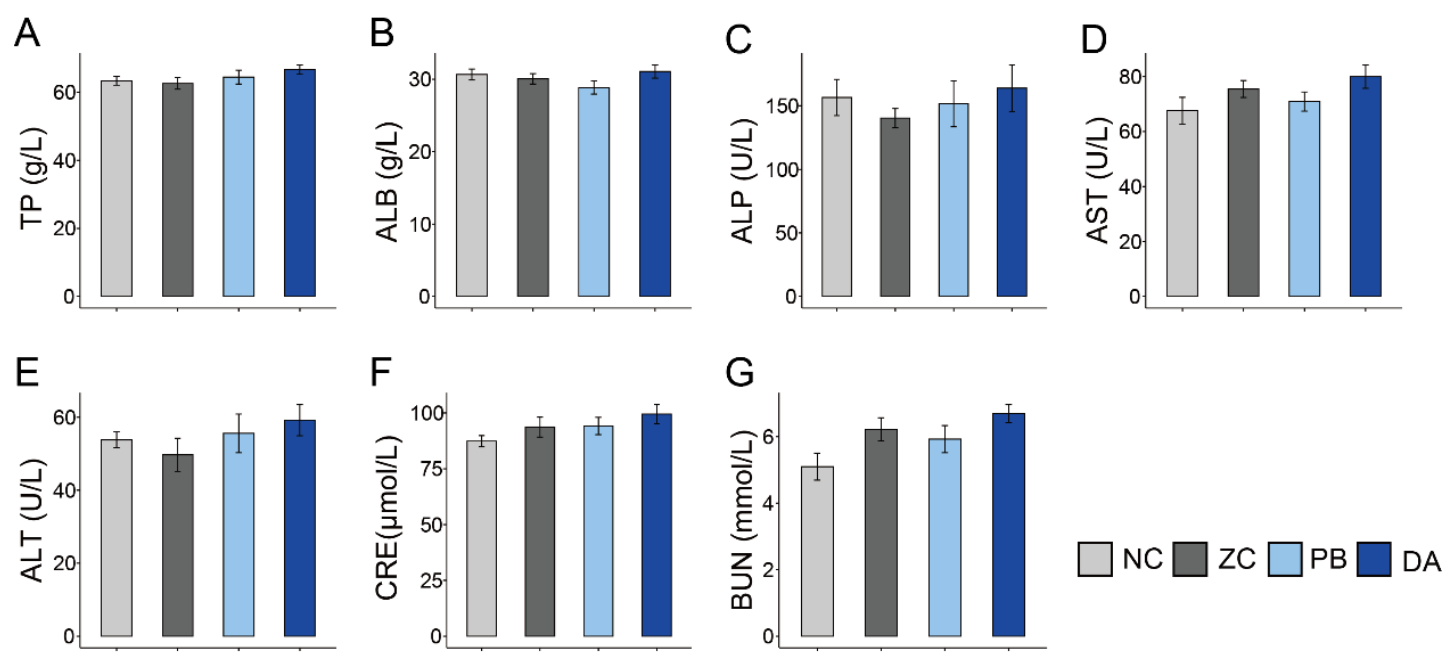

Figure 3. Comparison of serum levels of biochemical indicators among different treatment groups. (A) TP, total protein; (B) ALB, albumin; (C) ALP, alkaline phosphatase; (D) AST, aspartate aminotransferase; (E) ALT, alanine aminotransferase; (F) CRE, creatinine; (G) BUN, urea nitrogen. NC, normal control diet group; ZC, ZEN-contaminated group; PB, probiotic Bacillus subtilis group; DA, biodegradable Bacillus subtilis group.
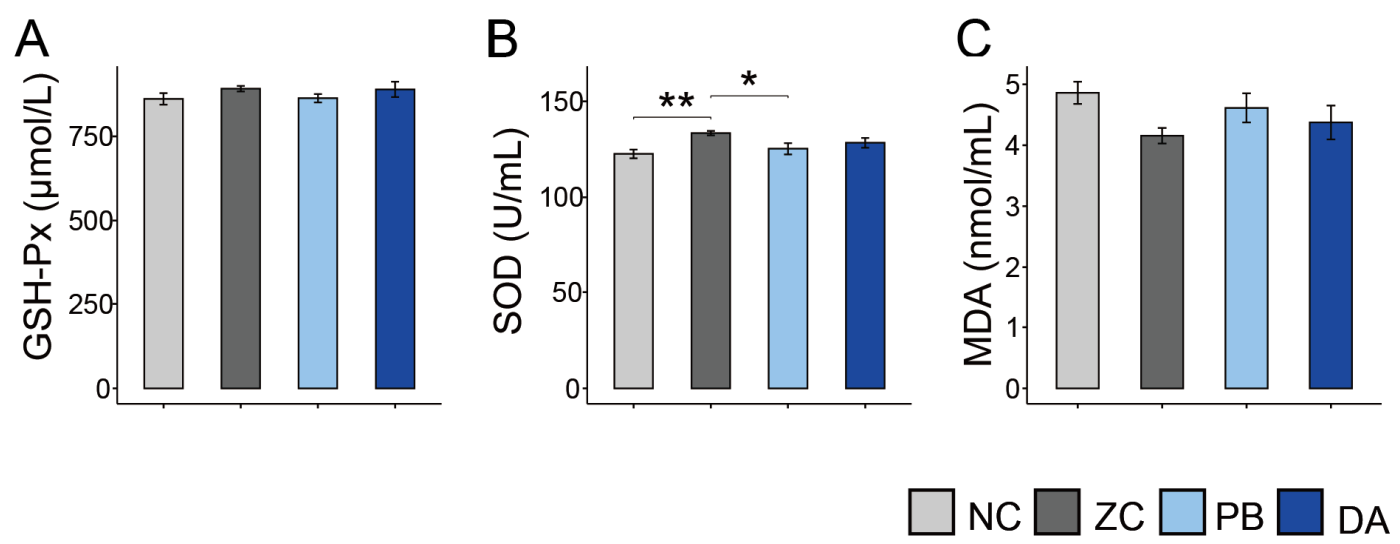

Figure 4. Comparison of serum levels of antioxidant parameters among different treatment groups. (A) GSH-Px, glutathione peroxidase; (B) SOD, superoxide dismutase; (C) MDA, malondialdehyde. NC, normal control diet group; ZC, ZENcontaminated group; PB, probiotic Bacillus subtilis group; DA, biodegradable Bacillus subtilis group. ${ }^{*}, p<0.05 ;{ }^{* *}, p<0.01$. 
As shown in Figure 5, the levels of immunoglobulin G (IgG) and $\operatorname{IgM}$ in the PB and DA groups were dramatically lower than that of the ZC group (Figure $5 B, C, p<0.05$ ). However, there was no significant difference in the levels of IgA (Figure $5 \mathrm{~A}, p<0.05$ ). In addition, the levels of pro-inflammatory factors (e.g., interleukin 2 (IL-2) and tumor necrosis factor- $\alpha(\mathrm{TNF} \alpha)$ ) in the serum of the ZC group was significantly higher than these of other groups (Figure 5E,G, $p<0.05$ ). Additionally, the DA group had the lowest level of IL-2 (Figure 5E, $p<0.05$ ). However, other pro-inflammatory factors (e.g., IL-1 $\beta$ and IL-6) were not significantly different (Figure 5D,F, $p>0.05$ ).

A

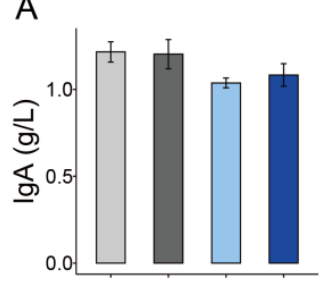

$\mathrm{E}$

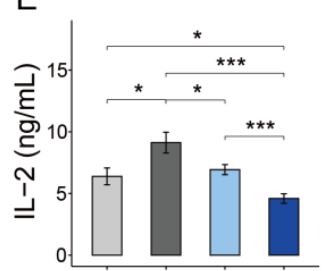

B

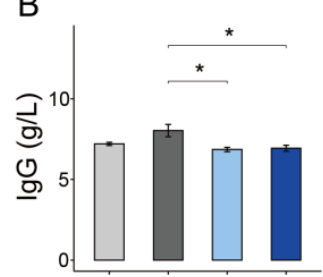

$\mathrm{F}$

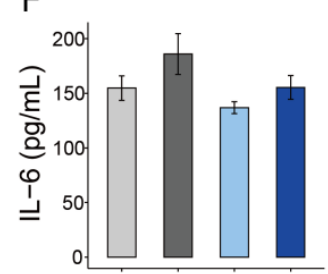

C

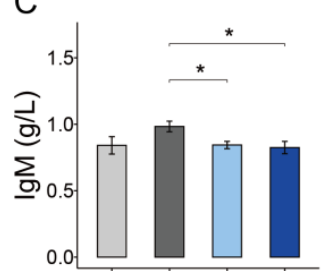

G

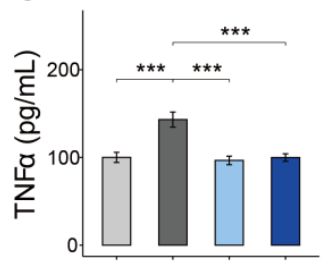

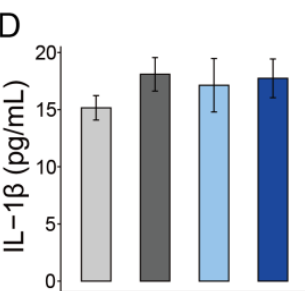

$\square \mathrm{NC} \square \mathrm{zC} \square \mathrm{PB} \square \mathrm{DA}$

Figure 5. Comparison of serum levels of immune and inflammatory parameters in different treatment groups. (A-C) IgA, immunoglobulin A; IgG, immunoglobulin G; IgM, immunoglobulin M; (D-G) IL-1 $\beta$, interleukin 1 $\beta$; IL-2, interleukin 2; IL-6, interleukin 6; TNF $\alpha$, tumor necrosis factor- $\alpha$; NC, normal control diet group; ZC, ZEN-contaminated group; PB, probiotic Bacillus subtilis group; DA, biodegradable Bacillus subtilis group. ${ }^{*}, p<0.05 ;{ }^{* * *}, p<0.001$.

\subsection{Serum Hormone Parameters}

The effects of diet supplemented with ZEN or Bacillus subtilis on the serum hormone of gilts were also shown in Figure 6. The level of estradiol (E2) in the NC and DA groups were significantly lower than that in the ZC groups (Figure 6C, $p<0.05$ ), while no significant difference in the levels of follicle-stimulating hormone (FSH) and luteinizing hormone $(\mathrm{LH})$ were observed in each treatment group (Figure 6A,B, $p>0.05$ ). In addition, the level of prolactin (PRL) in the ZC group was the highest among the four groups (Figure 6D, $p<0.05)$. There was no difference in the PRL levels of the PB and DA groups compared to the NC group (Figure 6D, $p>0.05$ ).

A

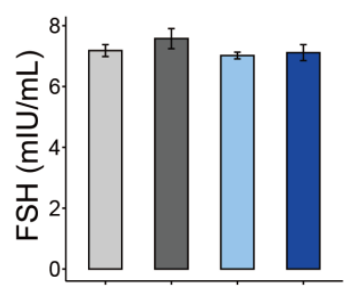

$\mathrm{B}$

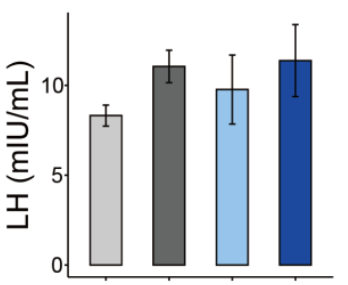

C

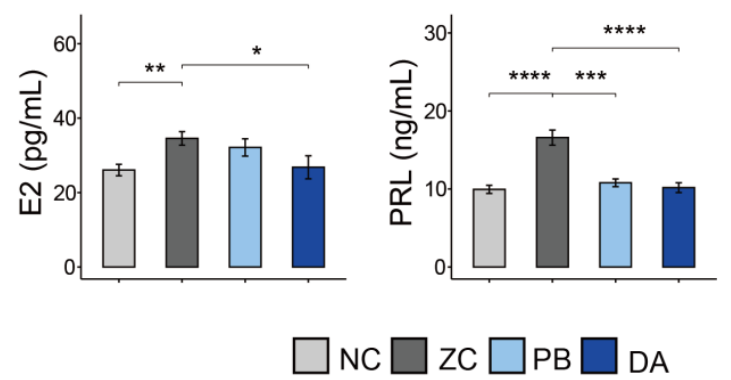

Figure 6. Comparison of serum levels of hormone parameters in different treatment groups. (A-D) FSH, follicle-stimulating hormone; LH, luteinizing hormone; E2, estradiol; PRL, prolactin. NC, normal control diet group; ZC, ZEN-contaminated group; PB, probiotic Bacillus subtilis group; DA, biodegradable Bacillus subtilis group. ${ }^{*}, p<0.05 ;{ }^{* *}, p<0.01 ;{ }^{* * *}, p<0.001$; $* * * *, p<0.0001$. 


\subsection{ZEN Residues}

As shown in Table 6, mildewed maize enormously increased the content of ZEN in feed and feces. The NC group had very low levels of ZEN in feed $(p=0.06)$ and fecal samples $(p<0.05)$. In contrast, the ZC group had the highest levels of ZEN in feed and fecal samples $(p<0.05)$. Surprisingly, the content of ZEN in feces of the DA group were dramatically lower than the ZC and PB groups $(p<0.05)$. Additionally, the ZEN content in feces of the PB group was nearly same to that of the ZC group $(p>0.05)$. Intriguingly, although the ratio of ZEN contents between feces to diet was not significantly among the four groups $(p>0.05)$, the value in the DA group was indeed half that of the remaining three groups. In this study, both ZEN and its metabolites ( $\alpha$-zearalanol $(\alpha-Z A L), \beta$-zearalanol ( $\beta$-ZAL), $\alpha$-zearalenol ( $\alpha$-ZOL), $\beta$-zearalenol $(\beta$-ZOL) and zearalanone $(\mathrm{ZAN}))$ were not found in serum samples.

Table 6. Content of ZEN in feed and fecal samples.

\begin{tabular}{|c|c|c|c|c|c|c|}
\hline Items & NC & $\mathrm{ZC}$ & PB & DA & SEM & $p$-Value \\
\hline Content of ZEN in diet, $\mu \mathrm{g} / \mathrm{kg}$ & 17.50 & 304.80 & 297.30 & 307.70 & 55.42 & 0.06 \\
\hline Content of ZEN in feces, $\mu \mathrm{g} / \mathrm{kg}$ & $7.23^{b}$ & $104.24^{\mathrm{a}}$ & $98.86^{\mathrm{a}}$ & $55.37^{\mathrm{b}}$ & 17.34 & 0.00 \\
\hline $\begin{array}{l}\text { Ratio of ZEN contents between } \\
\text { feces to diet, \% }\end{array}$ & 41.34 & 34.20 & 33.26 & 17.30 & 5.87 & 0.73 \\
\hline
\end{tabular}

NC, normal control diet group; ZC, ZEN-contaminated group; PB, probiotic Bacillus subtilis group; DA, biodegradable Bacillus subtilis group. Different superscript letters represent significant difference.

\section{Discussion}

The recent years have witnessed growing interests in finding practical and effective methods to detoxify ZEN in contaminated cereals and feeds [15-17]. Previous studies had been focused on mycotoxin adsorbents used to control mycotoxins in animal feed [18]. However, these adsorbents would contribute to environmental pollution as they transfer mycotoxins to surrounding areas [19]. Biodegradation of mycotoxins was considered as an efficient and environmentally protective method for the treatment of contaminated diets in the livestock production [11]. Previous studies have shown that some strains of Bacillus spp. were able to prevent the toxicity of ZEN [13,14], while it is not well known whether these effects were due to their probiotic or degradative capacities. Previous reports from our laboratory suggested biodegradable Bacillus subtilis ANSB01G could degrade $84.58 \%, 83.04 \%$ and $66.34 \%$ of ZEN in naturally contaminated maize, swine complete feed and dried distillers' grains with solubles, respectively [13]. For these reasons, it is worth comparing the ameliorative effects between probiotic and biodegradable Bacillus subtilis in a ZEN-contaminated diet. The persuasion of the present study was ensured by the similarity of Bacillus subtilis ANSB010 and ANSB01G on the habitat source and the bacteriostatic activity against common pathogenic bacteria, including E. coli, S. choleraesuis and S. aureus.

It has been reported that the presence of ZEN reduced feed consumption, caused a subsequent growth depression, and increased susceptibility to diseases [20,21]. Although we did not observe significant difference in gilts weight in the present study, there is a decreasing trend in ADFI among these groups. In line with previous reports [22], our study showed that the gilts fed on diets containing ZEN significantly increased F/G. In our study, the contaminated diet contained about $300 \mu \mathrm{g} / \mathrm{kg}$ of ZEN, leading to an increase in ZEN levels in the feces. Our data revealed that biodegradable Bacillus subtilis ANSB01G alleviated the toxicity while significantly decreasing the $\mathrm{F} / \mathrm{G}$, but probiotic Bacillus subtilis ANSB010 did not.

Previous study has indicated that vulva swelling is the main clinical symptom of ZEN-induced toxicity in mammals [23,24], which has an adverse impact on the reproductive system and the breeding performance. Similarly, our data also indicated that ZEN significantly caused an increase in vulva size. Previous research found that the mycotoxin biodegradation agent composed of Bacillus subtilis ANSB01G and Devosia sp. ANSB714 can effectively reduce the estrogenic swelling of the vulva caused by ZEN in immature gilts [25]. 
In this study, we also discovered that only biodegradable Bacillus subtilis ANSB01G mediates vulva swelling caused by ZEN. Some available evidence also demonstrated the obvious adverse effects of ZEN on the secretion of these hormones and productivity of animals [26]. In addition, similar results were reflected by a fluctuation in hormone levels. Although there were no significant difference in the levels of FSH and LH, ZEN diets markedly increased the level of E2 in the gilts. It was well known that ZEN is a competitive substrate for endogenous estrogens, binding to estrogen receptors and thereby having a damaging effect on the function of gonads [27]. A recent long-term (48 d) study found that low doses of ZEN $(20 \mu \mathrm{g} / \mathrm{kg} \mathrm{BW})$ induced changes in the concentrations of E2 levels in pre-pubertal gilts [28]. The addition of biodegradable Bacillus subtilis ANSB01G, in this study, restored serum E2 levels and modulated the function of gonads in gilts.

Van and his colleagues reported that ZEN ingestion partially induced oxidative stress in piglets, as it revealed an increased content of MDA and activity of SOD [29]. In addition, even low levels of ZEN $(246 \mathrm{ug} / \mathrm{kg})$ in the diet of gestation sows can lead to an increase in the level of serum MDA and cause cell apoptosis and moderate lesions of the liver, kidney, uterus, and ovary [30]. Importantly, our research showed that only biodegradable Bacillus subtilis ANSB01G reversed these increasing trends. However, no changes in the levels of antioxidant enzymes (e.g., GSH-Px and MDA) or SOD activity were observed in the serum of gilts. Moreover, we did not observe that diets treated with ZEN or both strains of Bacillus subtilis affected the serum biochemical indicators (e.g., ALB, AST, ALP and CRE). These data were inconsistent with the previous reports [31,32]. The difference in results might be attributed to the age difference of the animal model and different doses of ZEN contaminated. However, we noticed that ZEN increased IgG level in serum, but not in the levels of IgA and IgM. Gilts treated with biodegradable Bacillus subtilis ANSB01G and probiotic Bacillus subtilis ANSB010 decreased IgG levels in serum. IgG, IgM and IgA are the main components of immunoglobulins, of which the content of $\operatorname{IgG}$ is up to $70-75 \%$. These results suggested that ZEN has antigenic activity which stimulated the immune system of gilts, and both biodegradable and probiotic Bacillus subtilis have the capacity to recover the stimulation caused by ZEN.

Moreover, it has been reported that ZEN could increase the synthesis and expression of pro-inflammatory factors through JNK signaling pathway activation [6]. Currently, few studies have focused on the effects of ZEN on the modulation of inflammation in gilts. In the present study, ZEN in the feed significantly increased the levels of TNF $\alpha$ and IL-2, and had no effects on IL- $1 \beta$ and IL- 6 in serum. An increase in levels of TNF $\alpha$, one of the most powerful pro-inflammation factors, might generate a risk of a more severe inflammatory response [33]. The inflammatory response in this trial was similar to previous results from our lab that showed an enormous increase in inflammatory cytokines (e.g., IL-2, IL-8 and IL-10) [25]. The elevation of cytokines caused by ZEN would impair the erythroid progenitor and red blood cells [34], which revealed a potential cancerotoxic effect of ZEN. Biodegradable Bacillus subtilis ANSB01G mitigated the acute inflammatory response, confirming the reliability of a biodegradation approach in myotoxin degradation. In comparison, the lack effect of probiotic Bacillus subtilis ANSB010 on the elevated inflammatory response demonstrated that probiotic Bacillus subtilis had no effects on alleviating the toxicity of ZEN. Therefore, we concluded that biodegradable Bacillus subtilis was more protective against ZEN toxicosis in gilts than the probiotic Bacillus subtilis. Taken together, this study provided further evidence that the specific strain of Bacillus subtilis ANSB01G can alleviate the toxicity of ZEN, mainly due to its biodegradable capacity.

\section{Conclusions}

This study demonstrated that a feeding diet contaminated with ZEN of $300 \mu \mathrm{g} / \mathrm{kg}$ had a damaging impact on the growth performance, plasma immune function and hormone secretion of gilts. Although probiotic Bacillus subtilis ANSB010 and biodegradable Bacillus subtilis ANSB01G have similar antimicrobial capacities and alleviate inflammatory responses, only biodegradable Bacillus subtilis ANSB010 could regulate estrogen levels, 
relieve swelling of the vulva, and reduce the F/G and fecal ZEN residues. Hence, the biodegradable Bacillus subtilis ANSB01G used in this study is considered to have great and promising potential for biodegradation of mycotoxin in feed industrial applications.

\section{Materials and Methods}

\subsection{Source and Identification of Bacterial Strains}

The two strains of Bacillus subtilis used in this study were isolated from healthy broiler intestinal chyme and identified and characterized using a standard method described by Holt et al. [35]. Gram-staining was performed using the Gram staining kit (G1060, Solarbio, Beijing, China). Briefly, the bacteria were activated in Luria-Bertani (LB) medium at $37^{\circ} \mathrm{C}$ for $12 \mathrm{~h}$. Then, a $2.5 \mu \mathrm{L}$ sample was stained with crystal violet for $1 \mathrm{~min}$, mordanted with iodine solution $1 \mathrm{~min}$, decolorized for $30 \mathrm{~s}$, and counterstained with safranine for $1 \mathrm{~min}$. Spore-staining was performed using the Spore stain kit (G1133, Solarbio, Beijing, China). First, a $5 \mu \mathrm{L}$ sample, cultured in LB medium at $37^{\circ} \mathrm{C}$ for $48 \mathrm{~h}$, was stained in malachite green solution for $10 \mathrm{~min}$ and counterstained with safranine for $3 \mathrm{~min}$. Finally, the staining results were observed using a microscope. Then, DNA was extracted from the bacterial isolates using the Bacterial Genomic DNA kit (Beijing Zoman Biotechnology Co., Ltd., Beijing, China) according to the manufacturer's instructions. PCR amplification of $16 \mathrm{~S}$ rDNA was performed with the primers 27F (5'-AGAGTTTGATCMTGGCTCAG-3') and 1492R (5'-CGGTTACCTTGTTACGACTT-3'), and PCR products were purified and sequenced by Sangon Biotech (Beijing, China). Probiotic and biodegradable Bacillus subtilis were identified as strains of Bacillus subtilis named ANSB010 and ANSB01G, respectively. It has been shown that ANSB01G could degrade ZEN in naturally contaminated maize with high efficiency [13]. The Bacillus subtilis ANSB01G in our experiment was a domesticated strain based on the wild bacteria obtained by Lei et al. [13]. The method of microbial domestication was as follows: after activation, Bacillus subtilis ANSB01G was induced and cultured in a series of MRS mediums with gradually increasing concentrations of ZEN [36]. The efficiency of Bacillus subtilis ANSB01G in degrading ZEN was further improved after several domestications. The $16 \mathrm{~s}$ rDNA sequences of ANSB010 and domesticated ANSB01G are shown in the Supplementary Materials. A phylogenetic tree was drawn with the neighbor-joining method of 1000 bootstrap replications within Mega 5.0.

\subsection{Antibacterial Activity and ZEN Degradation Tests}

Selected indicated bacteria E. coli (No.10003), S. choleraesuis (No.21493) and S. aureus (No.10384) were purchased from the China Center of Industrial Culture Collection (CICC, Beijing, China). E. coli, S. choleraesuis and S. aureus were inoculated in MRS medium and incubated in MRS medium at $180 \mathrm{rpm}$ for $24 \mathrm{~h}$ at $37^{\circ} \mathrm{C}$. After diluting twice in a gradient, $200 \mu \mathrm{L}$ of the bacterial solution was added to MRS medium and spread evenly, then $\mathrm{z}$ sterilized Oxford cup was placed on the medium. Then, $200 \mu \mathrm{L}$ of the supernatant of the probiotic and biodegradable Bacillus subtilis solution was aspirated into an Oxford cup and the non-inoculated MRS medium was used as a control. After incubating at $37^{\circ} \mathrm{C}$ for $24 \mathrm{~h}$, the antibacterial circle diameter $(\mathrm{cm})$ was measured. For the ZEN degradation test, ZEN solution $(100 \mu \mathrm{L}, 2 \mu \mathrm{g} / \mathrm{mL})$ was added into the LB medium of ANSB010 and ANSB01G $\left(900 \mu \mathrm{L}, 3.0 \times 10^{8} \mathrm{CFU} / \mathrm{mL}\right)$ and incubated with shaking for $6 \mathrm{~h}, 24 \mathrm{~h}$, and $48 \mathrm{~h}$ at $37^{\circ} \mathrm{C}$ in the dark, followed by measurement of ZEN levels using the HPLC method.

\subsection{Animals and Experimental Treatments}

The animal experiments were conducted according to the animal welfare requirements and approved by the Animal Protocol Review Committee of the China Agriculture University (Beijing, China).

Thirty-six healthy gilts (Landrace $\times$ Yorkshire, average body weight $=64 \mathrm{~kg}$ ) were selected for the experiment. Then, these animals were randomly assigned to four treatments with nine replicates of one gilt per replicate for each group: (1) Normal control diet group (NC) fed the basal diet containing few ZEN (17.5 $\mu \mathrm{g} / \mathrm{kg}$ diet) by controlling the quality 
of maize; (2) ZEN-contaminated group (ZC) fed the contaminated diet containing an exceeded limit dose of ZEN (about $300 \mu \mathrm{g} / \mathrm{kg}$ diet) by replacing normal maize in the basal diet with moldy maize; (3) Probiotic agent group (PB) fed the ZC diet with added $5 \times 10^{9}$ $\mathrm{CFU} / \mathrm{kg}$ of probiotic Bacillus subtilis ANSB010; (4) Biodegradable agent group (DA) fed the ZC diet with added $5 \times 10^{9} \mathrm{CFU} / \mathrm{kg}$ of biodegradable Bacillus subtilis ANSB01G. The contaminated maize was purchased in 2018 from a small family farm in Henan Province, China. The Bacillus subtilis ANSB010 and ANSB01G were incubated in LB medium for $24 \mathrm{~h}$ at $37^{\circ} \mathrm{C}$, followed by drying at $65^{\circ} \mathrm{C}$, and then evenly mixed into the diets. Diets are formulated to meet or exceed nutrient requirements (Table 7) recommended by the National Research Council for replacement gilts (NRC, 2012). The experimental period lasts for $25 \mathrm{~d}$. The contaminated diets were prepared by replacing corn in the control with the naturally contaminated maize. During the supplementation period, all piglets were individually housed in temperature-controlled stainless steel metabolism pens $\left(25 \pm 2{ }^{\circ} \mathrm{C}\right)$, allowing free access to drinking water. Animal care and experimental procedures were in accordance with the guidelines of the National Institutes of Health Guide and the China Ministry of Agriculture for the care and use of laboratory animals.

Table 7. Ingredients and compositions of the basal diet, as fed basis.

\begin{tabular}{cccc}
\hline Ingredient & $\mathbf{\%}$ & $\begin{array}{c}\text { Nutrition } \\
\text { Component }\end{array}$ & Content $\mathbf{1}^{\mathbf{1}}$ \\
\hline $\begin{array}{c}\text { Maize } \\
\text { Soybean meal }\end{array}$ & 57.00 & DE, Kcal/Kg & 3100 \\
Wheat bran & 23.00 & Crude protein, $\%$ & 17.00 \\
Calcium & 16.00 & Calcium, \% & 0.76 \\
hydrophosphate & 1.00 & Total phosphorus, \% & 0.61 \\
Limestone & 1.05 & Non-phytate & 0.36 \\
Salt & 0.30 & phosphorus, \% & 0.85 \\
Threonine & 0.05 & Lysine, \% & 0.52 \\
Lysine $70 \%$ & 0.60 & Methionine, \% & 0.59 \\
Choline chloride & 0.12 & Threonine, \% & \\
Chlortetracycline & 0.05 & & \\
Compound-premix ${ }^{2}$ & 0.83 & & \\
Total & 100.00 & & \\
\hline
\end{tabular}

${ }^{1}$ The value is calculated. ${ }^{2}$ Supplied the following per kilogram of diet: vitamin A, $5000 \mathrm{IU}$; vitamin D3, $900 \mathrm{IU}$; vitamin E, $40 \mathrm{IU}$; vitamin K, $2.5 \mathrm{mg}$; vitamin B1, $1.5 \mathrm{mg}$; vitamin B2, $6.4 \mathrm{mg}$; vitamin B6, $2.5 \mathrm{mg}$; vitamin B12, 0.025 $\mathrm{mg}$; pantothenate, $20 \mathrm{mg}$; nicotinic acid, $30 \mathrm{mg}$; choline, $0.50 \mathrm{~g} ; \mathrm{Fe} 100 \mathrm{mg}$; Cu, $6 \mathrm{mg}$; Zn, $50 \mathrm{mg}$; Mn, $20 \mathrm{mg}$; Se, $0.30 \mathrm{mg}$; I, $0.24 \mathrm{mg}$.

\subsection{Growth Performances}

The initial body weight and terminal body weight were recorded. Moreover, ADG, ADFI, and F/G (ADFI/ADG) were calculated.

\subsection{Vulva Size Determination}

The vulva length (a), width (b) and height (h) were measured and recorded at $0,12 \mathrm{~d}$ and $24 \mathrm{~d}$. The determination of vulva area and volume was performed and results were calculated according to the method previously described by Zhao and his colleagues [14]. The area and volume of vulva are approximately elliptical and conical, respectively. Therefore, the area of the vulva is in accordance with the formula: $S=(\pi \times a \times b) / 4$; and the volume of the vulva is calculated with the equation: $\mathrm{V}=1 / 3 \times \mathrm{S} \times \mathrm{h}$.

\subsection{Serum Parametes}

Blood was collected from the marginal ear vein at the end of the experiment period. Then, the blood samples were centrifuged at $3000 \times g$ for $10 \mathrm{~min}$ to obtain serum for further analysis. The serum biochemical indicators including the TP, ALB, ALT, AST and ALP, CRE and BUN were measured with an automatic biochemical analyzer (Hitachi 7160, Hitachi High-Technologies Corporation, Tokyo, Japan). Immunoglobulin A (IgA), IgG and IgM 
were also determined with an automatic biochemical analyzer (Hitachi 7160). In addition, interleukin $1 \beta$ (IL-1 $\beta$ ), IL-2, IL-6 and TNF $\alpha$ were measured by an enzyme-linked immune sorbent assay (ELISA) kit (YuanMu Biotechnology, Shanghai, China). Serum SOD, GSH-Px and MDA were detected according to the instructions of the manufacturer using microplate test kits (Nanjing Jiancheng Bioengineering Institute, Nanjing, China). The concentrations of serum E2, FSH, LH and PRL were determined by radioimmunoassay (RIA). The serum samples were treated with radioactive-125I according to the instructions of the RIA kit (Beijing Kemei Biotechnology Co., Ltd., Beijing, China). Then, a GC-1200 radio-immunity gamma-counter (KeDa Innovation Co., Ltd., Hefei, China) was used to measure hormone concentrations. For each RIA, the intra- and inter-assay coefficients of variation were less than $15 \%$ and less than $10 \%$, respectively. Hormone concentration was determined according to each sample's level of radioactivity.

\subsection{Determination of ZEN in the Feed, Feces, Broth and Serum}

Two days before the end of the test, fresh fecal samples were taken to determine the content of ZEN in feces according to the description of Chinese certification GB/T 235042009 and Lei et al. [13]. For feed and feces samples, $50 \mathrm{~g}$ ground samples were extracted by acetonitrile-water $(70: 30, v / v, 200 \mathrm{~mL})$, followed by filtration with Whatman 4 filter paper. After dilution with PBS solution ( $\mathrm{PH}=7.40)$, the mixing solution were filtered through a micro-filter. A volume of $20 \mathrm{~mL}$ of suspension was passed through the immunoaffinity column (Femdetection FD-C21, Nanjing, China) at a flow rate of $1.0 \mathrm{~mL} / \mathrm{min}$ under gravity. After washing the column with distilled water, the ZEN was subsequently eluted with $2 \mathrm{~mL}$ of methanol into a centrifuge tube for HPLC analysis. For liquid medium, samples (1 mL) were extracted with acetonitrile $(9 \mathrm{~mL})$ at $180 \mathrm{rpm}$ for $2 \mathrm{~h}$. The mixed samples were filtered using glass fibre filter paper, and then collected for subsequent HPLC analysis. ZEN and its metabolites in serum were analyzed using the method described by Duca et al. [37]. Briefly, serum $(2 \mathrm{~mL})$ was mixed with buffer ammonium acetate solution $(8 \mathrm{~mL})$. The mixed solution was incubated with glucuronidase/arylsulfatase $(50 \mu \mathrm{L})$ at $37^{\circ} \mathrm{C}$ for $15 \mathrm{~h}$. After the samples were centrifuged at $5000 \mathrm{rpm}$ for $10 \mathrm{~min}$, the supernatant was passed through the immunoaffinity column. The column was then rinsed with $20 \mathrm{~mL}$ of ultrapure water. The analytes were then eluted with acetonitrile $(2 \mathrm{~mL})$. Subsequently, the solution was dried using a Speed Vac concentration system after which $200 \mu \mathrm{L}$ mobile phase was added. For HPLC analysis, $20 \mu \mathrm{L}$ sample solution was injected into the HPLC system. Separation was in a C18 column $(4.6 \mathrm{~mm} \times 150 \mathrm{~mm}, 5 \mu \mathrm{m}$; Thermo Fisher Scientific, Waltham, MA, USA) with mobile phase (water: acetonitrile, $50: 50, v / v$ ) at a flow rate of $1.0 \mathrm{~mL} / \mathrm{min}$. The analytes were detected by a fluorescence detector (Waters, Milford, MA, USA), excitation and emission wavelengths were 274 and $440 \mathrm{~nm}$, respectively. The retention time was 7-8 min.

\subsection{Statistical Analysis}

Data were analyzed statistically using SAS 9.4 software (SAS Institute Inc., Cary, $\mathrm{NC}, \mathrm{USA}$ ) and were presented as mean \pm SEM. The significance of difference between groups of gilts were analyzed by one-way ANOVA. A normality test (Shapiro-Wilk) was performed to determine normality before one-way ANOVA analysis. Differences were regarded as statistically significant at a probability of $p<0.05$, and $p$-values $<0.10$ were regard as a trend.

Supplementary Materials: The following are available online at https:/ /www.mdpi.com/article/10 .3390/toxins13120882/s1.

Author Contributions: W.S. and Y.L. (Yaojun Liu): conceptualization, conducted the animal experiments; methodology, writing — review and editing. X.Z. (Xinyue Zhang), X.Z. (Xiong Zhang), F.L. and J.C.: assisted with the experiments; X.R. and Y.L. (Yuanpei Lei): conducted the ZEN degradation test; L.Z. and C.J.: supervision; Q.M.: conceptualization, funding acquisition, writing—review and editing. All authors have read and agreed to the published version of the manuscript. 
Funding: This study was funded by the National Natural Science Foundation of China (Grant No. 31772637, 31301981), and the Special Fund for Agro-scientific Research in the Public Interest (201403047).

Institutional Review Board Statement: The study was conducted according to the guidelines of the Beijing Muncipality on the Review of Welfare and Ethics of Laboratory Animals, and approved by the China Agricultural University Animal Care and Use Committee (Approval No. Aw72011202-1-6).

Informed Consent Statement: Not applicable.

Data Availability Statement: Data are available from the first author.

Acknowledgments: The authors wish to thank the students who participated in our study and the workers of the Fengze Farm.

Conflicts of Interest: The authors declare no conflict of interest.

\section{References}

1. Kuiper-Goodman, T.; Scott, P.; Watanabe, H. Risk assessment of the mycotoxin zearalenone. Regul. Toxicol. Pharmacol. 1987, 7, 253-306. [CrossRef]

2. Zinedine, A.; Soriano, J.M.; Moltó, J.C.; Mañes, J. Review on the toxicity, occurrence, metabolism, detoxification, regulations and intake of zearalenone: An oestrogenic mycotoxin. Food Chem. Toxicol. 2007, 45, 1-18. [CrossRef] [PubMed]

3. Ma, R.; Zhang, L.; Liu, M.; Su, Y.-T.; Xie, W.-M.; Zhang, N.-Y.; Dai, J.-F.; Wang, Y.; Rajput, S.A.; Qi, D.-S.; et al. Individual and Combined Occurrence of Mycotoxins in Feed Ingredients and Complete Feeds in China. Toxins 2018, 10, 113. [CrossRef]

4. Döll, S.; Dänicke, S. The Fusarium toxins deoxynivalenol (DON) and zearalenone (ZON) in animal feeding. Prev. Vet. Med. 2011, 102, 132-145. [CrossRef] [PubMed]

5. Jiang, S.Z.; Yang, Z.B.; Yang, W.R.; Gao, J.; Liu, F.X.; Broomhead, J.; Chi, F. Effects of purified zearalenone on growth performance, organ size, serum metabolites, and oxidative stress in postweaning gilts1. J. Anim. Sci. 2011, 89, 3008-3015. [CrossRef]

6. Pistol, G.C.; Braicu, C.; Motiu, M.; Gras, M.A.; Marin, D.E.; Stancu, M.; Calin, L.; Israel-Roming, F.; Berindan-Neagoe, I.; Taranu, I. Zearalenone Mycotoxin Affects Immune Mediators, MAPK Signalling Molecules, Nuclear Receptors and Genome-Wide Gene Expression in Pig Spleen. PLoS ONE 2015, 10, e0127503. [CrossRef]

7. Awad, W.A.; Ghareeb, K.; Böhm, J.; Zentek, J. Decontamination and detoxification strategies for the Fusarium mycotoxin deoxynivalenol in animal feed and the effectiveness of microbial biodegradation. Food Addit. Contam. 2010, 27, 510-520. [CrossRef]

8. Rustom, I.Y.S. Aflatoxin in food and feed: Occurrence, legislation and inactivation by physical methods. Food Chem. 1997, 59, 57-67. [CrossRef]

9. Schatzmayr, G.; Zehner, F.; Täubel, M.; Schatzmayr, D.; Klimitsch, A.; Loibner, A.P.; Binder, E.M. Microbiologicals for deactivating mycotoxins. Mol. Nutr. Food Res. 2006, 50, 543-551. [CrossRef] [PubMed]

10. Jardon-Xicotencatl, S.; Díaz-Torres, R.; Marroquin-Cardona, A.; Villarreal-Barajas, T.; Méndez-Albores, A. Detoxification of Aflatoxin-Contaminated Maize by Neutral Electrolyzed Oxidizing Water. Toxins 2015, 7, 4294-4314. [CrossRef]

11. Jouany, J.P. Methods for preventing, decontaminating and minimizing the toxicity of mycotoxins in feeds. Anim. Feed. Sci. Technol. 2007, 137, 342-362. [CrossRef]

12. Doyle, M.P.; Applebaum, R.S.; Brackett, R.E.; Marth, E.H. Physical, Chemical and Biological Degradation of Mycotoxins in Foods and Agricultural Commodities. J. Food Prot. 1982, 45, 964-971. [CrossRef]

13. Lei, Y.; Zhao, L.; Ma, Q.; Zhang, J.Y.; Zhou, T.; Gao, C.; Ji, C. Degradation of zearalenone in swine feed and feed ingredients by Bacillus subtilis ANSB01G. World Mycotoxin J. 2014, 7, 143-151. [CrossRef]

14. Zhao, L.; Lei, Y.; Bao, Y.; Jia, R.; Ma, Q.; Zhang, J.; Chen, J.; Ji, C. Ameliorative effects ofBacillus subtilisANSB01G on zearalenone toxicosis in pre-pubertal female gilts. Food Addit. Contam. Part A 2014, 32, 617-625. [CrossRef] [PubMed]

15. Galvano, F.; Piva, A.; Ritieni, A.; Galvano, G. Dietary Strategies to Counteract the Effects of Mycotoxins: A Review. J. Food Prot. 2001, 64, 120-131. [CrossRef] [PubMed]

16. Binder, E.M. Managing the risk of mycotoxins in modern feed production. Anim. Feed. Sci. Technol. 2007, 133, 149-166. [CrossRef]

17. Zaki, M.M.; El-Midany, S.; Shaheen, H.; Rizzi, L. Mycotoxins in animals: Occurrence, effects, prevention and management. J. Toxicol. Environ. Health Sciences 2012, 4, 13-28. [CrossRef]

18. Huwig, A.; Freimund, S.; Käppeli, O.; Dutler, H. Mycotoxin detoxication of animal feed by different adsorbents. Toxicol. Lett. 2001, 122, 179-188. [CrossRef]

19. Kološová, A.; Stroka, J. Substances for reduction of the contamination of feed by mycotoxins: A review. World Mycotoxin J. 2011, 4 , 225-256. [CrossRef]

20. Swamy, H.V.L.N.; Smith, T.K.; Macdonald, E.J.; Karrow, N.A.; Woodward, B.; Boermans, H.J. Effects of feeding a blend of grains naturally contaminated with Fusarium mycotoxins on growth and immunological measurements of starter pigs, and the efficacy of a polymeric glucomannan mycotoxin adsorbent. J. Anim. Sci. 2003, 81, 2792-2803. [CrossRef] 
21. Pierzgalski, A.; Bryła, M.; Kanabus, J.; Modrzewska, M.; Podolska, G. Updated Review of the Toxicity of Selected Fusarium Toxins and Their Modified Forms. Toxins 2021, 13, 768. [CrossRef]

22. Cheng, Y.-H.; Weng, C.-F.; Chen, B.-J.; Chang, M.-H. Toxicity of different Fusarium mycotoxins on growth performance, immune responses and efficacy of a mycotoxin degrading enzyme in pigs. Anim. Res. 2006, 55, 579-590. [CrossRef]

23. Zhang, J.; Zheng, Y.; Tao, H.; Liu, J.; Zhao, P.; Yang, F.; Lv, Z.; Wang, J. Effects of Bacillus subtilis ZJ-2019-1 on Zearalenone Toxicosis in Female Gilts. Toxins 2021, 13, 788. [CrossRef]

24. Gao, X.; Xiao, Z.; Li, C.; Zhang, J.; Zhu, L.; Sun, L.; Zhang, N.; Khalil, M.M.; Rajput, S.A.; Qi, D. Prenatal exposure to zearalenone disrupts reproductive potential and development via hormone-related genes in male rats. Food Chem. Toxicol. 2018, 116, 11-19. [CrossRef] [PubMed]

25. Shi, D.; Zhou, J.; Zhao, L.; Rong, X.; Fan, Y.; Hamid, H.; Li, W.; Ji, C.; Ma, Q. Alleviation of mycotoxin biodegradation agent on zearalenone and deoxynivalenol toxicosis in immature gilts. J. Anim. Sci. Biotechnol. 2018, 9, 42. [CrossRef] [PubMed]

26. Hassan, A.A.; Rashid, M.; Koratum, K.M. Effect of aflatoxin B1, zearalenone and ochratoxin A on some hormones related to fertility in male rats. Life Sci. J. 2010, 7, 64-72.

27. Silva, J.R.; Hurk, R.V.D.; de Matos, M.H.T.; dos Santos, R.R.; Pessoa, C.; de Moraes, M.O.; de Figueiredo, J.R. Influences of FSH and EGF on primordial follicles during in vitro culture of caprine ovarian cortical tissue. Theriogenology 2004, 61, 1691-1704. [CrossRef] [PubMed]

28. Zielonka, L.; Gajęcka, M.; Lisieska-Żołnierczyk, S.; Dąbrowski, M.; Gajęcki, M.T. The Effect of Different Doses of Zearalenone in Feed on the Bioavailability of Zearalenone and Alpha-Zearalenol, and the Concentrations of Estradiol and Testosterone in the Peripheral Blood of Pre-Pubertal Gilts. Toxins 2020, 12, 144. [CrossRef] [PubMed]

29. Van Le Thanh, B.; Lemay, M.; Bastien, A.; Lapointe, J.; Lessard, M.; Chorfi, Y.; Guay, F. The potential effects of antioxidant feed additives in mitigating the adverse effects of corn naturally contaminated with Fusarium mycotoxins on antioxidant systems in the intestinal mucosa, plasma, and liver in weaned pigs. Mycotoxin Res. 2016, 32, 99-116. [CrossRef]

30. Zhou, J.; Ao, X.; Lei, Y.; Ji, C.; Ma, Q. Bacillus subtilis ANSB01G culture alleviates oxidative stress and cell apoptosis induced by dietary zearalenone in first-parity gestation sows. Anim. Nutr. 2020, 6, 372-378. [CrossRef]

31. Long, M.; Yang, S.; Zhang, W.; Zhang, Y.; Li, P.; Guo, Y.; Wang, Y.; Chen, X.; He, J. The Influence of Selenium Yeast on Hematological, Biochemical and Reproductive Hormone Level Changes in Kunming Mice Following Acute Exposure to Zearalenone. Biol. Trace Element Res. 2016, 174, 362-368. [CrossRef] [PubMed]

32. Su, Y.; Sun, Y.; Ju, D.; Chang, S.; Shi, B.; Shan, A. The detoxification effect of vitamin C on zearalenone toxicity in piglets. Ecotoxicol. Environ. Saf. 2018, 158, 284-292. [CrossRef] [PubMed]

33. Ben Salah-Abbès, J.; Belgacem, H.; Ezzdini, K.; Abdel-Wahhab, M.A.; Abbès, S. Zearalenone nephrotoxicity: DNA fragmentation, apoptotic gene expression and oxidative stress protected by Lactobacillus plantarum MON03. Toxicon 2020, 175, 28-35. [CrossRef]

34. Tiemann, U.; Brüssow, K.-P.; Dannenberger, D.; Jonas, L.; Pöhland, R.; Jäger, K.; Dänicke, S.; Hagemann, E. The effect of feeding a diet naturally contaminated with deoxynivalenol (DON) and zearalenone (ZON) on the spleen and liver of sow and fetus from day 35 to 70 of gestation. Toxicol. Lett. 2008, 179, 113-117. [CrossRef]

35. Cowan, S.T. Bergey's Manual of Determinative Bacteriology. Nature 1948, 162, 833. [CrossRef]

36. Ma, Q.G.; Zhao, L.H.; Ji, C.; Zhang, J.Y.; Li, M.L.; Zheng, R. A Strain Domestication Method for Improving the Degradation Efficiency of Mycotoxin-Degrading Bacteria. Patent CN10804 8385A, 2018.

37. Duca, R.C.; Bravin, F.; Delaforge, M.; Vladescu, L.; Badea, I.A.; Criste, R.D. Development of a New HPLC Method Used for Determination of Zearalenone and Its Metabolites in Broiler Samples. Influence of Zearalenone on the Nutritional Properties of Broiler Meat. J. Agric. Food Chem. 2009, 57, 10497-10504. [CrossRef] 